\title{
Soluble guanylyl cyclase activation promotes angiogenesis
} Anastasia Pyriochou*, Chris Polytarchou, Evangelia Papadimitriou and
Andreas Papapetropoulos

\author{
Address: Laboratory of Molecular Pharmacology, Department of Pharmacy, University of Patras, Greece \\ Email: Anastasia Pyriochou* - apapapet@upatras.gr \\ * Corresponding author
}

from 2nd International Conference of cGMP Generators, Effectors and Therapeutic Implications

Potsdam, Germany, 10-12 June, 2005

Published: 16 June 2005

BMC Pharmacology 2005, 5(SuppI I):P46 doi:I0.1 186/I47I-22 I0-5-SI-P46

Soluble guanylyl cyclase (sGC) is an $\alpha / \beta$ heterodimeric protein that is activated by nitric oxide (NO), yielding increased intracellular levels of cGMP. Evidence in the literature suggests that endogenously produced $\mathrm{NO}$, as well as NO-donors, stimulate angiogenesis by increasing endothelial cell (EC) proliferation, migration and organization of EC into vascular networks. Moreover, NO mediates many of the angiogenic actions of vascular endothelial growth factor. Although sGC is expressed in the endothelium, its autocrine actions have not been thoroughly studied. Herein, we have investigated the role of sGC in neo-vessel formation using the chicken chorioallantoic membrane (CAM) model of angiogenesis. Initially, we confirmed the presence of sGC subunits in the CAM during the time of maximal angiogenesis. Moreover, when cells transfected with a luciferase reporter gene under the control of the sGC $\alpha 1$ promoter were placed on the CAM, we observed that expression of this sGC subunit was higher at day 12 than day 6 (angiogenesis reaches its maximum at day 12 in the CAM). The existence of catalytically active sGC in the CAM was confirmed through the increase in CGMP tissue content after exposure to a NO donor (sodium nitroprusside). To study the role of sGC in angiogenesis we used several pharmacological agents that modulate cGMP levels in tissues (ODQ, a sGC inhibitor; BAY 41-2272, an NO-independent/heme-dependent activator of sGC; and zaprinast, a phosphodiesterase V inhibitor). ODQ inhibited angiogenesis in a dose-dependent manner, while BAY 41-2272 and zaprinast promoted neovascularization. In line with the in vivo data, ODQ inhibited VEGF-stimulated endothelial cell migration and organization of HUVEC into tube-like structures on matrigel. We conclude that sGC activation promotes neovessel formation. 